\section{TB Skin Test Conversion Rates Reduced by Expanded TB Control Program}

Researchers at Emory University School of Medicine and Grady Memorial Hospital in Atlanta recently reported a significant decrease in healthcare worker $(\mathrm{HCW})$ tuberculin skin test (TST) conversion rates (over a 2-year period, $2.7 \%$ to $0.6 \%$ ) following the implementation of specific tuberculosis (TB) control measures. The TB control measures, which were instituted after a documented outbreak on two wards of the hospital, consisted of: 1) expanded respiratory isolation policy; 2) increased TB surveillance; 3) expanded HCW education; 4) increased frequency of mandatory TST (from annual to every 6 months). Interim engineering controls (pending renovation of the hospital) consisted of conversion of rooms without recirculated air to negative pressure with outside exhaust (but without six air exchanges per hour). In addition, a particulate respirator was introduced for use by HCWs. The researchers concluded that infection control measures, which consisted primarily of administrative controls in this hospital, were effective in reducing nosocomial transmission of TB to HCWs.

FROM: Blumbergh M, Watkins D, Hunter M, et al. Prevention of nosocomial transmission of TB by administrative controls. Presented at the 1994 Infectious Disease Society of America Annual Meeting. Orlando, Florida. Abstract 109.

\section{Outbreakof $P$ aeruginosa Infection Associated with Healthcare Worker Dermatitis}

Researchers at Geisinger Medical Center in Danville, Pennsylvania, recently reported seven cases of Pseudomonas aeruginosa infection (4 conjunctivitis, 2 primary bacteremias, 1 trachea-bronchitis) in a neonatal intensive care unit (NICU). Environmental cultures of the NICU were negative for the organism. Epidemiologic typing of the five available outbreak strains suggested that three were identical. Investigation revealed that nurse $\mathrm{A}$, who had exposure to all three cases with identical strains, had weeping dermatitis and was culture positive for $\mathbf{P}$ aeruginosa. Follow-up cultures of this HCW after treatment for dermatitis were negative for $\mathbf{P}$ aeruginosa.

FROM: Bross JE, Dahlmann M, Bourbeau P. NICU outbreak of Pseudomonas aeruginosa infection associated with healthcare worker dermatitis. Presented at the 1994 Infectious Disease Society of America Annual Meeting; Orlando, Florida. Abstract 110.

\section{Healthcare Worker Carriage of MRSA Linked to Pet Dog}

Dr. Christopher Cefai and colleagues from the United Kingdom reported two cases of nosocomial methicillin- resistant Staphylococcus aureus (MRSA) colonization in the intensive care unit (ICU) linked to a male staff nurse with nasal colonization. The spouse, a nurse working on another ward, also was found to be a nasal carrier of MRSA. The husband and wife were treated in accordance with practices in the United Kingdom. The healthcare workers were negative for MRSA 3 weeks after their treatment with nasal mupirocin and triclosan washes.

When another patient was found to be colonized with the same organism, screening identified the same nurse and his wife to be recolonized. An investigation revealed that the nurses' dog had an eye infection for several weeks; eye and nose swabs grew MRSA of the identical phage type. Treatment of the husband, wife, and dog with nasal mupirocin and triclosan washes successfully eliminated carriage of MRSA.

Staphylococci commonly are carried by animals but tend to be host-adapted varieties. Staphylococcus intermedius is the most common isolate from dogs, but human strains may be isolated. An outbreak of MRSA associated with a heavily colonized ward cat has been reported.

FROM: Cefai C, Ashurst S, Owens C. Human Carriage of methicillin-resistant Staphylococcus aureus linked with pet dog. Lancet August 20, 1994;344:539-540.

\section{High Prevalence of Candida Species on Hands of HCWs}

Researchers conducted a culture survey of more than 1,600 personnel in surgical (SICU) and neonatal intensive care units (NICU) to determine the presence of Candida colonization. Candida species were recovered from the hands of 15\% of HCWs in San Antonio, 19\% in Iowa City, 25\% in Atlanta, 39\% in New York, and 54\% in Portland. The rate of hand colonization in HCW in the SICU was $39 \%$ compared with $29 \%$ in the NICU. The most common species recovered was Candidaparapsilosis (46\%) followed by Candida albicans (15\%). The rate of carriage by occupation was $30 \%$ among nurses, 33\% among respiratory therapists, and 29\% among physicians. The authors concluded that hands may serve as a reservoir for transmission of these microorganisms to high-risk, critically ill patients and suggests the importance of handwashing.

FROM: Rangel-Frausto MS, Martin MA, Saiman L, et al. High prevalence of Candida spp. on hands of healthcare workers in surgical and neonatal intensive care units: a multicenter study. Presented at the 34th Interscience Conference on Antimicrobial Agents and Chemotherapy; October 4-7, 1994; Orlando, Florida. Abstract J106.

\footnotetext{
Additional news items in this issue: Joint Commission Approves Format of Quality Performance Reports for Healthcare Organizations (page 741), CDC Releases Draft Guideline for Isolation Precautions in Hospitals (page 744), Infectious Disease Specialists Did Not Wash Hands When Leaving Restroom (page 756), CDC Releases Final TB Guidelines (page 763).
} 